the tissues affected by the syphilitic neoplasm. The bromid in the combination may be of service in correcting vasomotor perturbations which probably exist in early nerve syphilis.

It is not my custom to advocate special preparations or formulas, but there is another preparation which I do not hesitate to recommend as a tonic and alterative in syphilis, especially where nerve disturbance exists. The preparation known as the three chlorids of mercury, iron and arsenic, (Renz and Henry's formula) has in my hands proved a reliable addition to the therapy of syphilis. It has the merit of elegance and definite dosage.

The question now arises, How long shall this treatment be continued? As a matter of principle, I believe that any course of treatment of less than three years duration is open to criticism. I am especially inclined to be dogmatic in regard to cases presenting early nerve or brain symptoms.

I have been much impressed by some of $\mathrm{Mr}$. Hutchinson's cases of nerve syphilis. It appeared to me that some of them might have been avoided by proper management. Hutchinson's treatment is largely symptomatic. If at the end of a six months' course the symptoms have disappeared, treatment is stopped, to be resumed only on the recurrence of symptoms. Steady systematic treatment by mercury, iodids and gold, is the sole assurance of safety for the syphilitic.

As for the results of this plan I have only this to say: After some years of experience with syphilis, I have rarely seen of late, visceral, bone, nerve, or brain involvement in cases in which my instructions have been carried out to the letter. Cases occasionally arise in which a tonic stimulant becomes necessary. Under such circumstances, I have found the wine and fluid extract of coca of great service.

Anemia is sometimes a very important factor in early nerve syphilis. Such cases demand iron and strychnia. The iron may sometimes be combined with the mercury in the form of the pil. duo (mercurial pill with exiccated sulphate of iron). Some cases may require alcoholic stimulants in small amount.

Great care should be exhibited in the management of debilitated syphilitics, else harm may be done by our specifics.

\section{A SET OF UTERINE DILATORS.}

\section{BY HENRY T. BYFORD, M.D.} [From the Trausactions of the Chicago Gyuecological society,

I have here a set of uterine dilators, designed for the purpose of keeping the cervix moderately dilated

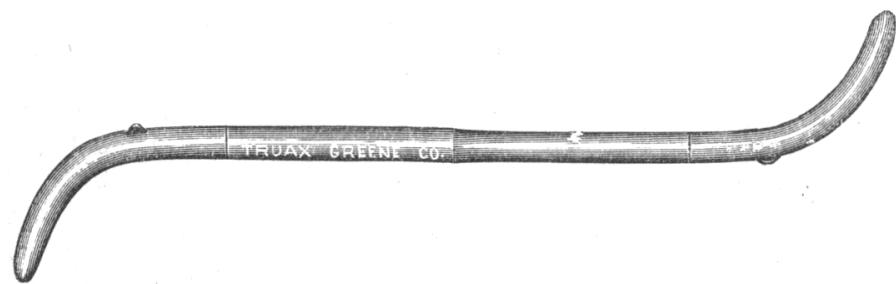

and thus maintaining uterine drainage in cases of endometritis with imperfectly developed or contracted cervices. These are stiff in the center, but will bend quite easily on the end. They can be used in a retroverted or an anteverted uterus. In my office practice
I use the two smaller sizes, and the larger ones after curettage to keep a poorly developed cervix dilated until the tendency to contract passes off. It is passed through the internal os twice a week, for a few times, and later once a week until the dilatation has been maintained for three or four months. The vaginal fornices should be cleansed with a 5 per cent. solution of carbolic acid before they are introduced and an intra-uterine application of a 25 per cent. solution of ichthyol in glycerin be made after their withdrawal.

\section{SOCIETY PROCEEDINGS.}

Proceedings of the First Meeting of the American Academy of Railway Surgeons.

Held at the Grand Pacific Hotel, Chicago, Ill., Nov. 9 and 10, 18.94. (Continued from page 212.)

Fifth Session, Saturday, November 10, 2 P.m.

Chairman Dr. H. J. Maynard read a letter from Dr. M. Cavana, Surgeon N. Y. P. \& O. R'y, Oneida, N. Y., expressing his regrets that on account of serious sickness in his family he was unable to be present but wished the Academy Godspeed.

The Academy then went in to executive session to consider the applications of Drs. C. B. Parker, Surgeon, L. S. \& M. S. R'y, Cleveland, Ohio: R. H. Taylor, Surgeon, Central R'y of Georgia, Griffin, Ga.; Geo.W. Crile, Surgeon, C. C. C. \& St. L. R'y, Cleveland, Ohio; J. G. Davis, Chief Surgeon L. N. A.\& C. R'y, Chicago, Ill.; A. D. Bevan, Chief Surgeon, Iowa Central R'y, Chicago, Ill.

The ballot having been cast it was found that all of the gentlemen named had been elected to fellowship.

\section{THE USE OF HORSE HAIR IN SURGERY.}

BY C. M. DANIELS, M.D.

CHIEF SURGEON W, N. Y. \& P. R'Y, BUFFALO, N. Y.

In presenting this subject I lay no claim to originality in the use of horse hair in surgical practice as it was suggested to me ten years ago ; but having used it extensively during the period mentioned, and not having seen the subject referred to but once in surgical literature, I venture to present this paper which will certainly be commended for its brevity.

For many years, surgeons bave been faithfully searching for the ideal suture, one that would be non-irritating, small in caliber, easily adjusted and afford the closest approximation of the skin layers, and this with especial reference to wounds of the face or exposed parts where the avoidance of scars, more or less unsightly, is a great desideratum.

Catgut, silk and silkworm gut have long been used, but in the first we have a rapidity of softening and relaxation that will not infrequently result in a gaping wound. In the second, the resulting "stitch marks" which are frequently long in disappearing added to the occasional irritation, and in the silkworm gut the rigidity of the suture it. self makes each undesirable in superficial wounds and all usually require dressings or coverings held by bandages which are both disagreeable and cumbersome, especially when the patient is able to be about or upon the street. I speak with special reference to those wounds which in themselves are not serious enough to confine the patient, this being the class of cases where I feel free to recommend horse hair sutures and upon which I note the following points :

1. Instead of a large or medium sized needle as required with even small strands of catgut, silk or silkworm gut, with the horse hair a small eye needle, curved or straight, according to the preference of the surgeon, can be used and thus minimize the size of each puncture of the skin.

2. Horse hair, when prepared as I will suggest later, has a firmness that will give support to the skin and very materially aid in keeping in approximation the edges of the same, and more nearly resemble silkworm gut in its action in the tissue.

3. Where wounds are both lacerated and contused, with 